\title{
Whose rings around Neptune?
}

\author{
What seems to be an authentic and interesting discovery of an incomplete ring around the planet \\ Neptune seems certain to be published piecemeal for lack of agreement among those concerned.
}

ReADers will be puzzled by the tentative explanation of the partial ring around Neptune provided on page 544 of this issue by Dr J. Lissauer of the University of California, Berkeley, chiefly because they have no reason to believe that there is such a structure around this distant planet. Surely, readers will ask, it will be time enough for Dr Lissauer to produce his explanation when there is evidence to suggest the need for one. And why should this apparently pointless explanation begin with the flat unsubstantiated declaration that "an incomplete arc system has recently been discovered around Neptune"?

Dr Lissauer should not be judged too harshly. Evidence for a partial ring system around Neptune does indeed exist. Roughly six months ago, an article giving a full and interesting account of the discovery was submitted to Nature for publication, was accepted for publication and would long since have been published had not those responsible for the collection of the evidence fallen out over the wording of the paper. All the authors have throughout expressed distress at being caught up in such a childish business yet have been unable to reconcile their differences.

What follows is an account of the circumstances leading to this sad state of affairs, from which two lessons may be learned. First, there may be occasions when pride takes precedence over the principle that science is an open process, in which publication is a duty owed to the rest of the community. Second, there are some outside the community who will ask whether science can be as deserving of public support as journals such as this repeatedly insist.

One of the prime movers in the discovery of the ring around Neptune appears to have been Professor André Brahic, from the University of Paris and the Observatoire de Paris, who has a long-standing interest in planetary ring systems. Brahic was the first author (among eight) of a manuscript "Occultation detection of a neptunian ring-like arc" which reached the Nature office at the end of May this year. The other authors quoted were W.B. Hubbard and F. Vilas from the University of Arizona, who have access to the Inter-American Observatory at Cerro Tololo in Chile, together with L.R. Elicer, a member of the observatory's staff; two colleagues of Brahic's from Paris, B. Sicardy and F. Roques; Patrice Bouchet, a staff member at the European Southern
Observatory (ESO), also in Chile, and two European astronomers, Jean Manfroid from the University of Liege and $R$. Haefner from the Max Planck Institute of Astrophysics at Munich.

The origins of the dispute among the authors can be traced to the night of 22 July 1984, when there was a nearoccultation of Neptune of a bright red star in Sagittarius, then visible from the two Chilean observatories. Brahic and his colleagues in Paris, who seem long to have suspected that Neptune may have a ring system, seem to have taken the initiative in drawing attention to this event. Ironically, Brahic had applied for observing time at ESO on the night in question, but had been given time to look at the rings of Uranus instead. Robbed of this chance, and with the good offices of Bouchet, Brahic seems at second-hand to have persuaded Haefner and Manfroid, the intended occupants of two telescopes at ESO, to turn away from their planned project to look at the occultation by Neptune instead.

In the event, Haefner and Manfroid found that there was indeed a brief second-long interruption of the infrared radiation from the distant star as it crossed an invisible circular orbit roughly 77,000 $\mathrm{km}$ nearly 3.0 radii from the centre of Neptune. One curious feature of the observations was that there was no corresponding dip in the radiation intensity as the distant star crossed the same circular orbit at its supposed second interception of it, whence the notion that, if there is a ring around Neptune, it is incomplete.

A similar sequence of events was found at Cerro Tololo, $100 \mathrm{~km}$ away in the Andes. Elicer began his observations later in the night, just a few minutes before the expected near-occultation and in some haste, to judge from the circumstance that it is not known what filter had been interposed in one of the two infrared channels. But the results are decisive and essentially the same as those found at the ESO site. Again there was a single interruption of the radiation, lasting about a second but no corresponding dip at the supposed second interception.

It is common-ground that these observations can only mean that there is absorbing material in one part of a supposedly circular orbit about Neptune, but that the material does not extend uniformly around the orbit. Brahic has rightly all along insisted that only coordinated observations at different sites can lend credibility to such fleeting events. (Clouds, electrical disturbances or passing flocks of birds could be held responsible for a brief interruption of radiation at a single site.) But with a well coordinated pair of observations in hand, Brahic was able to go back to previous attempts to find occulting material around Neptune. The pair of Andean observations last July, from sites separated by $100 \mathrm{~km}$, show that the absorbing material around Neptune must be at least $100 \mathrm{~km}$ in extent. The time for which radiation was interrupted suggests an object $15 \mathrm{~km}$ across.

So, now there is an interesting and quite novel phenomenon to be explained, a partial planetary ring. If it had been possible to publish the data on which the inference is based, people other than Lissauer would have been stimulated to bend their energies to this interesting problem. But why are the data still under lock and key?

Several things have gone wrong. The ESO observers, Haefner and Manfroid, have been offended by what they consider to have been unfair publicity overwhelming their contributions, a press release from the University of Arizona relayed in semi-popular journals such as $S k y$ and Telescope, for example. An article by Brahic in La Recherche last June seems to have given particular offence, although all the intended authors of the original Nature manuscript are duly listed in the margin. Haefner and Manfroid have published their data in the German-language journal Stern und Weltraum.

Much of the difficulty turns on the question of who "owns" the data collected at ESO, the observers who collected it or Brahic who drew attention to the occultation and to whom the data tapes were sent by prior arrangement? And what is to be done with data which, as in this case, are more interesting when combined with other people's than when published on their own?

The saddest feature of this development is that, whoever owns some of the data, there is a sense in which they belong to the scientific community as a whole. The upshot is that an important and even urgent set of data will not now be published quickly, as it should have been. The reputation of astronomers for good sense will have been undermined. Even the reputation of science as a whole will have been damaged - let us hope not irreparably. 